\title{
Standards for Smart Manufacturing: A review
}

\author{
Yuqian Lu, Huiyue Huang, Chao Liu, and Xun Xu
}

\begin{abstract}
Manufacturing is becoming smart with capabilities of self-awareness, autonomous decision-making, and adaptive excitation and collaboration. Standardization is a crucial enabler for achieving the required intelligence for smart manufacturing. Though a large number of efforts have been made to the development of manufacturing standards, there is still a significant research gap to be fulfilled. This paper reviews the landscape of existing standards in the context of smart manufacturing and offers guidance on the selection of the standards for different smart manufacturing applications.
\end{abstract}

\section{INTRODUCTION}

Today's manufacturers compete in a dynamic marketplace that demands excellence in quality and service, agility in production, and short response time to changing markets [1]. Manufacturing is shifting to a decentralized collaboration environment with a high-level uncertainty in market demands, business processes, and production facilities. Future manufacturing requires the pervasive application of smart Information and Communication Technologies (ICT) to create knowledge embedded production facilities, with predictive decision-making capabilities to ensure maximum throughput, excellent performance and smooth collaboration [2].

Over the past few years, international initiatives have collaboratively advocated a new generation of manufacturing - smart manufacturing [3-5]. Smart manufacturing is a fully-integrated, collaborative manufacturing ecosystem that responds in real time to meet changing demands and conditions in the factory, in the supply chain network, and customer needs. The United States has progressively devised its strategy for advanced manufacturing [6,7] since 2011 to enhance U.S. innovation and industrial competitiveness by facilitating the adoption of smart manufacturing. Germany initialized Industrie 4.0 initiative with the goal of efficient and low-cost production to maintain its leading role in future manufacturing.

Though a huge amount of global investment [8] on smart manufacturing technologies has generated some promising research outcomes for smart manufacturing applications [9], there are significant research gaps [4] to be addressed, and standardization is one of them. Recognizing the significance of standards for smart manufacturing, standard development bodies, such as International Standard Organization (ISO) and The American Society of Mechanical Engineers (ASME) have

*Research supported by China Scholarship Council.

Yuqian Lu is with the University of Auckland, Auckland, 1010 New Zealand (phone: +64 9923 1584; fax: +64 9373 7479; e-mail: yuqian.lu@auckland.ac.nz).

Huiyue Huang is with the University of Auckland, Auckland, 1010 New Zealand (e-mail: hhua920@aucklanduni.ac.nz).

Chao Liu is with the University of Auckland, Auckland, 1010 New Zealand (e-mail: cliu810@aucklanduni.ac.nz).

Xun Xu is with the University of Auckland, Auckland, 1010 New Zealand (e-mail: xun.xu@auckland.ac.nz). been revitalizing relevant standards. For example, ISO released ISO 10303 AP242 in 2014 to enable managed model-based 3D engineering [10]. A new standard (ISO/AWI 23247) targeting digital twin manufacturing framework is under development [11]. The standard is proposed to provide requirements and reference architecture for the realization of Digital Twin manufacturing, which is a trend of smart manufacturing. Moreover, community-based open standards, such as MTConnect [12] and OPC Unified Architecture (OPC UA) [13] have significantly improved the technological base for developing smart manufacturing solutions.

With an overwhelming portfolio of industry standards targeting smart manufacturing applications, there is a need to review the current standard landscape for smart manufacturing and provide guidance on how to use these standards properly. This paper summaries the latest standards for developing smart manufacturing solutions and reviews the reported research outcomes that use these standards to deliver to the vision of smart manufacturing. The rest of this paper is organized as follows. Section II briefly introduces the vision of smart manufacturing and highlights the role of standards in smart manufacturing. Current standard landscape for smart manufacturing is summarized in Section III. Section IV concludes the paper.

\section{STANDARDiZATION IN SMART MANUfactURING}

\section{A. Definition and Characters of Smart Manufacturing}

There are multiple definitions of smart manufacturing available coming from several different agencies, such as the Department of Energy (DoE) and the National Institute of Standards and Technology (NIST) in the United States. All these definitions highlight the use of ICT and advanced data analytics to improve manufacturing operations at all levels from shop-floor, through factory level to supply chain. Smart manufacturing offers tremendous potential for the manufacturing industry. Machines, systems, products, ICT systems, and people can be progressively connected over the Internet, creating a production network in which information carriers communicate with each other and exchange data and information in near real time.

\section{B. Significance of Standards}

Standardization is an essential requirement for integrating systems and processes and enabling collaborations between them. Different components can only work together if cross-manufacturer standards are established. Likewise, different companies can only collaborate with each other if engineering standards are adopted. There is a consensus that standardization is one of the top challenges for implementing smart manufacturing [14].

Given the collaborative and integrated nature of smart manufacturing, the standardization of architectures, data exchange formats, semantics, and interfaces are critical to 
maximizing business outputs between different technologies and solutions in smart manufacturing [15]. The focus of international standardization initiatives should, therefore, be placed on interoperable interfaces between smart manufacturing systems and the establishment of open standards. Standards need to be developed to declare the cooperation mechanisms and information to be exchanged for intra-company automation and inter-company integration [4]. Similarly, NIST highlights that there is a lack of open standards-based technologies that enable smart manufacturing systems to communicate, interact, exchange information, make decisions, and respond to faults [16].

\section{Smart Manufacturing Standard Dimensions}

The standardization activities taking place in the field of smart manufacturing are extremely dynamic, with several international initiatives working on smart manufacturing related standards. According to DIN - German Institute for Standardization [17], there are more than 300 standards related to smart manufacturing. All these dynamics make it impossible to systematically identify the ultimate list of recommended standards for smart manufacturing. Several studies so far have investigated the landscape of standards for smart manufacturing with distinct focuses and recommendations $[18,19]$.

This paper primarily focuses on the standards for enabling systems integration, with an emphasis on representative standards for integrated product development process and smart factory management.

\section{Standards FOR SMART PRODUCT LIFECYCLE MANAGEMENT}

Smart manufacturing requires distributed manufacturing businesses to work together to fulfill a highly customized product development need. A typical scenario is 'Design anywhere, build anywhere'. To achieve this, manufacturing companies and related software packages need to be capable of exchanging product data throughout the product development lifecycle without any interoperability issue.

Fig. 1 shows a timeline-based depiction of standards for different stages of product lifecycle. The plethora of standards in this space makes it challenging to coalesce a common vision across an organization. In this work, the discussion focuses on a collection of standards that can easily talk to each other with minimal interoperability risks to enable streamlined product data exchange between stages in a product lifecycle.

\section{A. Standards for Product Data Exchange}

ISO 10303, commonly known as STEP, is an international standard designed to exchange product data between CAD systems with a neutral data structure. It completed a major development of STEP AP242 in 2014, as shown in Fig. 2, for 'Managed Model Based 3D Engineering' by merging AP203 and AP204 with a focus on the representation of 3D model data, geometric tolerance, and Product Manufacturing Information (PMI) to enable global design and manufacturing collaboration [10].

STEP AP242 data model enables design and manufacturing companies to collaborate smoothly by exchanging machine-readable product design specifications between systems and companies, without the need for interpreting 2D drawings. A significant achievement of STEP AP242 is its support for semantic PMI that can make manufacturing systems smart [21]. The Geometric Dimensions \& Tolerances (GD\&T) data through AP 242 can be automatically consumed by downstream applications such as Computer Aided Process Planning (CAPP), Computer Aided Inspection (CAI), Computer Aided Tolerance Systems (CATS), and Coordinate Measuring Machines (CMM) [22].

\section{B. Standards for Manufacturing}

Standards for planning and execution of manufacturing is also integral to smart manufacturing. In this section, we view manufacturing in the narrow sense as the step to convert raw material to final product based on product specification. In the era of smart manufacturing, manufacturing focuses on 'batch size of 1' production, where a collection of fabrication methods, such as Numerical Control (NC) machining, additive manufacturing, and robotic machining work together. Interoperability between manufacturing systems is required to achieve flexible organization of manufacturing activities under changing conditions [23], and manufacturing equipment needs to be capable of interpreting manufacturing requirements from a Computer Aided Design (CAD) file at the semantic level and generating adaptive manufacturing strategies.

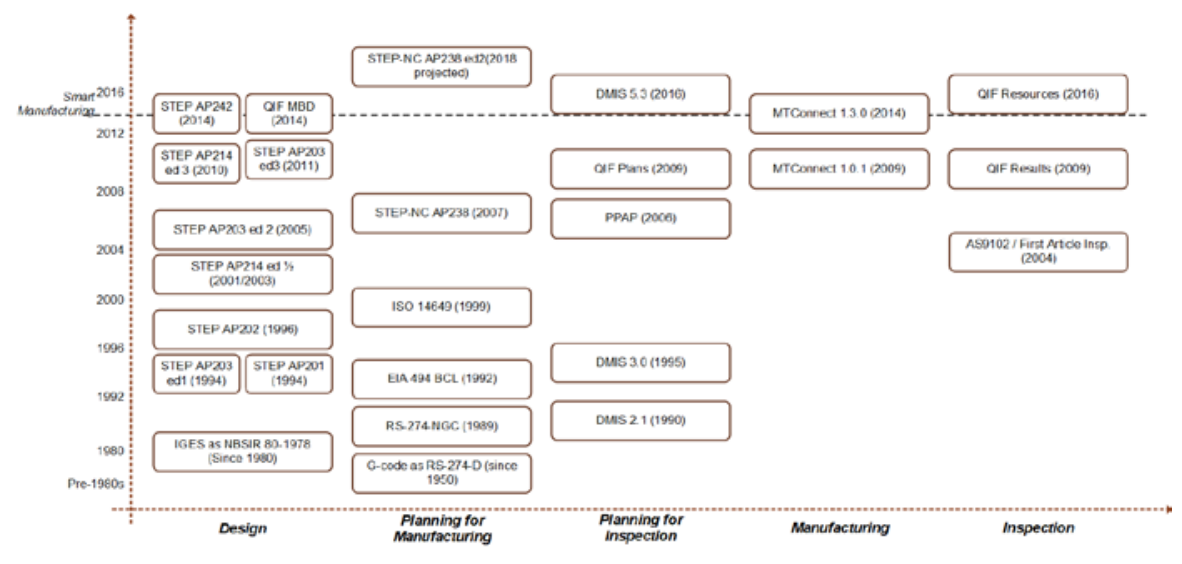

Figure 1. Timeline-based depiction of standards for different product lifecycle stages (adapted from [20]) 


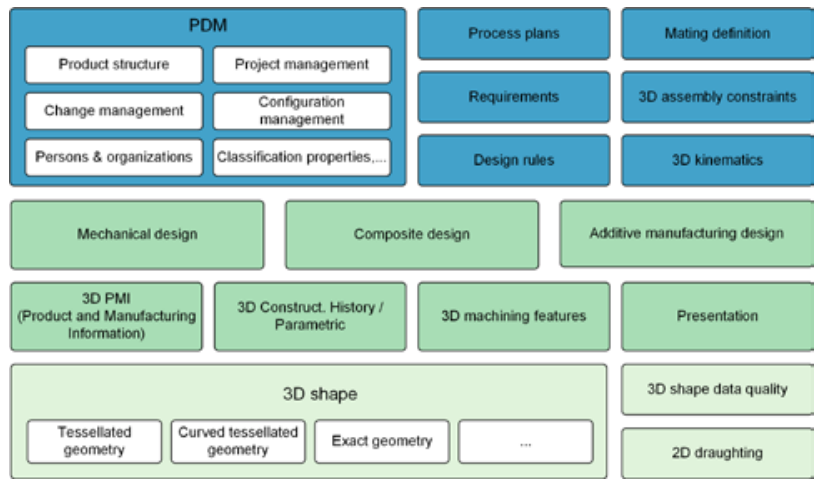

Figure 2. The high-level scope of ISO 10303 AP242

As a result, ISO 14649 and ISO 10303-238, also known as STEP-NC, are proposed to replace the RS274D (ISO 6983) G and $\mathrm{M}$ code. STEP-NC allows manufacturing organizations to seamlessly share machining information between machines. Unlike $\mathrm{G}$ and $\mathrm{M}$ code which contains only 'how-to-do' information, STEP-NC provides 'what-to-do' information to intelligent machine tools instead, giving enough flexibility to machine tools to interpret 'how-to-do' instructions adaptive to the local machining conditions. The shift of interpreting local machining instructions into machine controller maximizes the interoperability among distributed machine tools.

To this end, many studies have been conducted to achieve STEP-NC compliant manufacturing with applications in milling, turning, electro-erosion, industrial robotics, and additive manufacturing. In summary, STEP-NC compliant manufacturing operates at three intelligence level. The first level uses STEP-NC as a data carrier in the traditional $\mathrm{CAD} / \mathrm{CAM} / \mathrm{CNC}$ chain and converts a STEP-NC file to a machine-dependent NC code for machine execution [24]. This method allows easy integration with existing Computer Numerical Control (CNC) systems that reads G-code but does not fully get the designed benefits of STEP-NC. The second level of an intelligent controller can interpret STEP-NC file as native machine control data structures to drive fabrication [25]. The third level is adaptive CNC controller being capable of optimizing and executing machining in real-time by considering in-process machine condition. An adaptive CNC controller can fully understand a product's design intent and its quality requirement and devise an optimal manufacturing strategy according to the real-time local manufacturing environment.

Smart manufacturing needs an adaptive $\mathrm{CNC}$ controller that can directly take STEP-NC file and can communicate the as-built product model back to $\mathrm{CAD} / \mathrm{CAM}$ system. Fig. 3 depicts the STEP-NC complaint CAD/CAM/CNC chain, enabling interoperable design and manufacturing in a distributed design and manufacturing scenario.

\section{Standards for Process Monitoring}

Digital manufacturing depends on data from a diverse set of industrial equipment on the factory floor. Uniform, robust communications are part of the necessary infrastructure for modern business systems and analysis and decision-making. A widely-used standard to enable network-based equipment communication is MTConnect, which was developed for promoting the integration of $\mathrm{CNC}$ machines [12]. Another important standard to promote remote connectivity in the manufacturing environment is the OPC-UA [13]. This standard was developed by the OPC Foundation and emerged as an evolution of the classic OPC to represent a platform-independent interoperability standard in the exchange of data between the shop-floor and the enterprise. While MTConnect facilitates the connection of machine tools and other manufacturing equipment linked with a network for gathering data, OPC-UA promotes the needed interoperability for data communication throughout the plant [26].

\section{- $\quad$ MTConnect}

MTConnect is an open-source, royalty-free, and read-only standard that offers a semantic vocabulary for manufacturing equipment to provide structured, contextualized data with no proprietary format [27]. There are five fundamental components in a typical MTConnect application, including device, adapter, agent, network and client (Fig. 4).

MTConnect defines a hierarchical information model for machine tools. The information model represents the logical structure of a machine tool, including the components, the available data and the relationships among them. MTConnect defines the data at the source by providing a standard dictionary for manufacturing data. Specific modeling rules for the commonly used components and data in the machine tools are defined as eXtensible Markup Language (XML) Schemas. This feature significantly reduces the effort and time needed for building the information model and enhances the usability of the MTConnect-compliant applications.

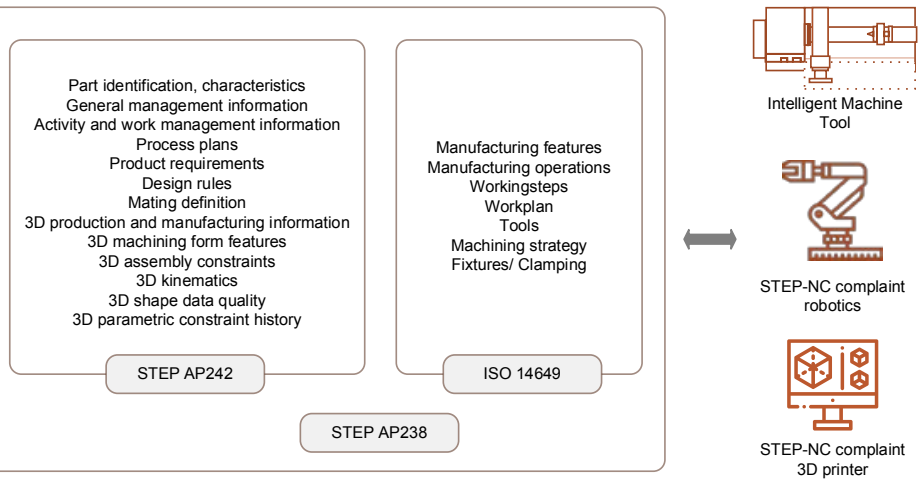

Figure 3. Information flow in a STEP-NC compliant manufacturing chain 


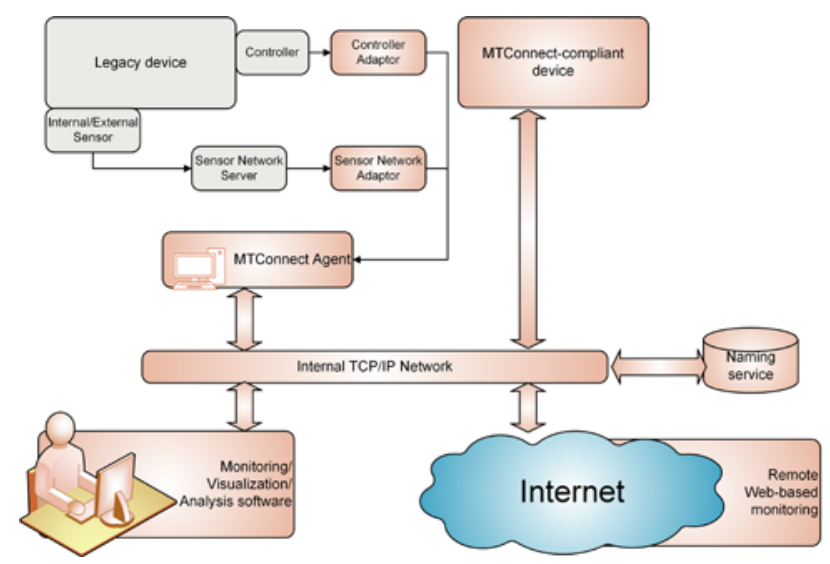

Figure 4. MTConnect signal flow from device to application (adapted from [28])

Research on the integration of MTConnect into process and equipment monitoring has been extensively studied. Edrington et al. [29] proposed a web-based machine monitoring system that provides data collection, analysis, and machine event notification for MTConnect compatible machines. MTConnect-based system monitoring is also used for retrieving in-process parts data to quickly generate machining quality reports [30]. Previous research also demonstrated a much wider application of MTConnect towards smart manufacturing, such as sustainable machining [31], machining simulation [32], cloud manufacturing [33], intelligent maintenance [34], and cyber-physical machine tools [35].

\section{- $\quad$ OPC UA}

OPC UA [13] is another open standard that specifies information exchange for industrial communication particularly on devices within machines, between machines and from machines to systems. OPC UA was the recommended industrial communication standard in the Reference Architecture Model Industry 4.0 (RAMI 4.0) [36]. It provides both communication protocol and information modeling method, allowing easy modeling and development of digital twins of manufacturing equipment in the cyberspace.

OPC UA defines a vendor and protocol independent client-server architecture based on standard web technologies that assure interoperability. Fig. 5 shows an example of OPC UA implementation at different levels of the automation pyramid, which proves that OPC UA enables data exchange through the field-level devices, the shop floor control systems, the enterprise applications as well as the cloud-based services.

One of the most important features of OPC UA is the possibility to define information models for virtually any device and system. Unlike MTConnect, OPC UA does not define a data dictionary, it defines an object-based Address Space Model [IEC 62541-3] as a meta-model that provides a standard way for servers to represent objects to clients instead. Based on this metamodel, vendors and developers can model their complex data as domain-specific information models to take advantage of the service-oriented architecture of OPC UA.

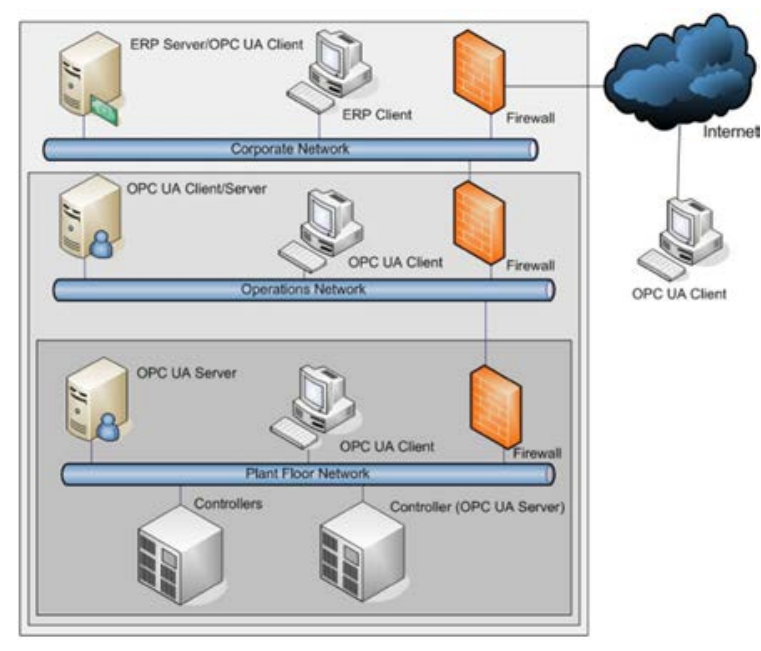

Figure 5. OPC UA implementation in the automation pyramid (adapted from [28])

Research on the implementation of OPC UA in process monitoring and control has been extensively studied. Ayatollahi et al. [37] developed an OPC UA-based semantic communication interface to control a machine tool remotely. Their results proved that OPC UA enhances the connectivity of robot tended machine tools. Various mechatronic functions of the machine tool and the robot are modeled as complex services in the OPC UA server to enhance the flexibility of the manufacturing cells. OPC UA is considered as a key enabler for Cyber-Physical Production Systems (CPPS). Schlechtendahl et al. [38] proposed a holistic approach to integrating existing production systems to the Industry 4.0 environment, in which OPC UA was validated as a critical enabler for discovering existing resources and enabling data communication through cloud-based gateways.

\section{- MTConnect versus OPC UA}

Though both MTConnect and OPC UA are considered as the future standard for communication and interfaces for smart manufacturing, there are enough differences. The main differences between MTConnect and OPC UA are summarized in Table I.

In order to improve the interoperability and consistency between these two standards, OPC Foundation and MTConnect Institute released a companion specification called MTConnect-OPC UA in 2012, providing a mechanism for OPC UA and MTConnect to collaborate and extend the reach of existing manufacturing data exchange standards [40].

\section{Standards for Smart Inspection}

Product inspection is an integral part of the product development process that verifies product quality on-site at various stages of the production process, from after receiving the raw materials to pre-shipping. At the current stage, inspection activities are mostly performed either as off-line inspection or on-line post-process inspection. However, an on-line in-process inspection that integrates with the product fabrication process is necessary for the production of highly customized products at small batches. 
TABLE I. MAIN DIFFERENCE BETWEEN MTCONNECT AND OPC UA (ADAPTED FROM [39])

\begin{tabular}{|c|c|c|}
\hline & MTConnect & OPC UA \\
\hline $\begin{array}{l}\text { Standard } \\
\text { domain }\end{array}$ & Machine tools & Any system \\
\hline $\begin{array}{l}\text { Information } \\
\text { modeling } \\
\text { method }\end{array}$ & $\begin{array}{l}\text { Structure of } \\
\text { information model is } \\
\text { defined as XML } \\
\text { schema in standard }\end{array}$ & $\begin{array}{l}\text { Generic and flexible; } \\
\text { users can design their own } \\
\text { information models and } \\
\text { data types }\end{array}$ \\
\hline Extensibility & $\begin{array}{l}\text { Relatively low; can } \\
\text { only be achieved } \\
\text { through the extension } \\
\text { of the standard }\end{array}$ & $\begin{array}{l}\text { High; any information } \\
\text { model can be built upon } \\
\text { the metamodel }\end{array}$ \\
\hline $\begin{array}{l}\text { Definitions of } \\
\text { data }\end{array}$ & $\begin{array}{l}\text { A detailed data } \\
\text { dictionary in the field } \\
\text { of machine tools is } \\
\text { concisely defined }\end{array}$ & $\begin{array}{l}\text { No domain-specific data } \\
\text { defined in the standard }\end{array}$ \\
\hline $\begin{array}{l}\text { Message } \\
\text { encoding }\end{array}$ & MTConnect XML & UA Binary and UA XML \\
\hline Security & $\begin{array}{l}\text { Low priority; not } \\
\text { integrated with the } \\
\text { standard since it is } \\
\text { read-only }\end{array}$ & $\begin{array}{l}\text { High priority; integrated } \\
\text { with each layer of the } \\
\text { communication stacks }\end{array}$ \\
\hline $\begin{array}{l}\text { Communication } \\
\text { ability }\end{array}$ & Read-only, one way & $\begin{array}{l}\text { Read and } \\
\text { bidirectional }\end{array}$ \\
\hline $\begin{array}{l}\text { Application } \\
\text { domain }\end{array}$ & Monitoring & Monitoring and control \\
\hline
\end{tabular}

The Quality Information Framework (QIF) is an ANSI (the American National Standards Institute) standard that defines an integrated set of XML information models to enable the productive exchange of metrology data throughout the entire manufacturing quality measurement process [41]. As shown in Fig. 6, a QIF-compliant metrology process starts with the generation of CAD and PMI data exported as QIF Model-Based Design (MBD) product model. Quality planning systems import the product model and generate the measurement plans according to the quality requirements and manufacturing processes. The inspection resources rules are considered at this stage. Programming systems then import the measurement plans to create Dimensional Measurement
Equipment (DME) specific programs. Dimensional measurement equipment executes the programs and evaluates the measurement results. At the end, analysis systems import single part results and generate the analysis of multiple part batches as QIF statistics data.

The research of using QIF standards across the product lifecycle is still in its infancy with just some theoretical discussions on harnessing QIF data into the product design and manufacturing processes [42]. Proctor, Franaszek, and Michaloski pointed out that exchanging manufacturing quality information is critical to the production optimization for smart manufacturing. They examined the requirements for exchanging product tolerance information in robotics manufacturing applications and mapped the requirements with existing standards such as STEP/STEP-NC and QIF [43]. Michaloski et al. [44] investigated the possibility of gathering continuous quality inspection results during the product machining process via integrating QIF standard with MTConnect, which provided a feasible solution for collecting and representing necessary process data and quality measurement data at the same time.

\section{CONCLUSION}

This paper reviews existing standards available for communication, data exchange, and integration alongside different stages of the product lifecycle in the context of smart manufacturing. Though these standards have shown some promising application scenarios to enable smart manufacturing, there is a strong need to review and upgrade as required to match the expectations and requirements of smart manufacturing. Meanwhile, there are information barriers among existing standards due to their different formulation background. Future work needs to focus on the feasibility, interoperability, and implementation analysis of existing standards for smart manufacturing.

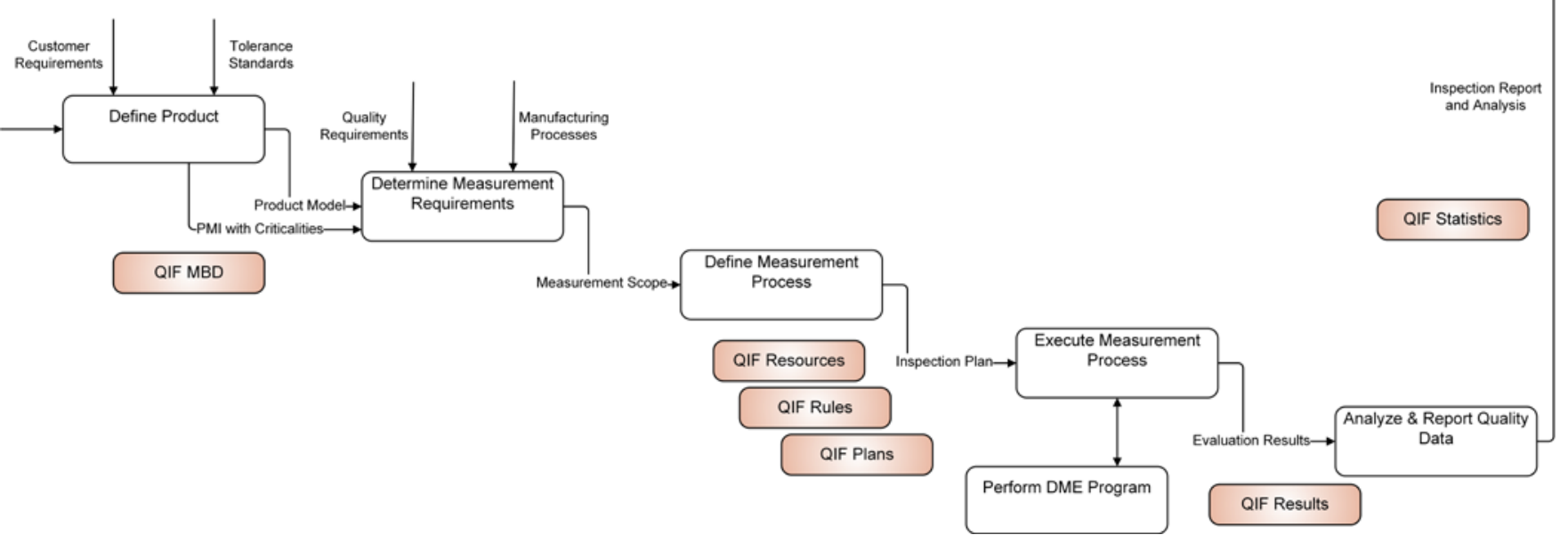

Figure 6. Integrated metrology process with QIF standard

\section{REFERENCES}

[1] L. Wang and A. J. Shih, "Challenges in smart manufacturing," Journal of Manufacturing Systems, vol. 40, p. 1, 2016.

[2] J. Davis, T. Edgar, J. Porter, J. Bernaden, and M. Sarli, "Smart manufacturing, manufacturing intelligence and demand-dynamic performance," Computers \& Chemical Engineering, vol. 47, pp. 145-156, 2012.

[3] S. M. L. Coalition, "Implementing 21st century smart manufacturing," in Workshop summary report, 2011.

[4] H. Kagermann, J. Helbig, A. Hellinger, and W. Wahlster, Recommendations for implementing the strategic initiative INDUSTRIE 4.0: Securing the future of German manufacturing 
industry; final report of the Industrie 4.0 Working Group. Forschungsunion, 2013.

[5] L. Li, "China's manufacturing locus in 2025: With a comparison of 'Made-in-China 2025' and 'Industry 4.0,'” Technological Forecasting and Social Change, vol. 135, pp. 66-74, 2018.

[6] A. Anderson, "Report to the President on Ensuring American Leadership in Advanced Manufacturing.," Executive office of the President, 2011.

[7] P. C. of A. on Science and Technology (US), Report to the President on Capturing Domestic Competitive Advantage in Advanced Manufacturing. Executive Office of the President, President's Council of Advisors on Science and Technology, 2012.

[8] R. Geissbauer, J. Vedso, and S. Schrauf, "Industry 4.0: Building the digital enterprise," Available: https://www. pwc. $\mathrm{com} / \mathrm{gx} / \mathrm{en} /$ industries/industries-4.0/landing-page/industry-4.0-buildin g-your-digital-enterprise-april-2016. pdf, 2016. [Accessed April 7, 2018].

[9] L. Monostori et al., "Cyber-physical systems in manufacturing," CIRP Annals, vol. 65, no. 2, pp. 621-641, 2016.

[10] ISO 10303-242, Industrial Automation Systems and Integration-Product Data Representation and Exchange-Part 242: Application Protocol: Managed Model-Based 3D Engineering. International Organization for Standardization (ISO) Geneva, Switzerland, 2014.

[11] ISO/AWI 23247, Digital Twin manufacturing framework. Available: https://www.iso.org/standard/75066.html [Accessed April 8, 2018].

[12] MTConnect Institute. MTConnect n.d. Available: http://www.mtconnect.org/ [Accessed February 25, 2018].

[13] OPC Foundation. Unified Architecture - OPC Foundation n.d. Available: https://opcfoundation.org/about/opc-technologies/opc-ua/ [Accessed February 25, 2018].

[14] R. Glatz, B. Diegner, and W. Dorst, "Tendenzumfrage der Plattform Industrie 4.0-Ergebnisse Januar-Februar 2013,” Plattform Industrie 4.0., 2013.

[15] H. Kagermann, R. Anderl, J. Gausemeier, G. Schuh, and W. Wahlster, Industrie 4.0 in a Global Context: strategies for cooperating with international partners. Herbert Utz Verlag, 2016.

[16] K. D. Thompson, "Smart Manufacturing Operations Planning and Control Program," Available: https://www.nist.gov/programs-projects/smart-manufacturing-operatio ns-planning-and-control-program [Accessed April 7, 2018].

[17] Industry 4.0 Standards. DIN - Ger Inst Stand. Available: https://www.din.de/en/innovation-and-research/industry-4-0/standards [Accessed June 28, 2018].

[18] Adolph, Lars. "German Standardization Roadmap: Industry 4.0." Version 2. Berlin: DIN eV. $2016 . \quad$ Available: https://sci40.com/files/assets sci40.com/pdf/german-standardization-r oadmap-industry-4-0-version-2-data.pdf [Accessed February 3, 2019].

[19] National Smart Manufacturing Standards Architecture Construction Guidance. 2015.

[20] W. Z. Bernstein, T. D. Hedberg Jr, M. Helu, and A. B. Feeney, "Contextualising manufacturing data for lifecycle decision-making," Int. J. Product Lifecycle Management, vol. 10, no. 4, 2017.

[21] A. B. Feeney, S. P. Frechette, and V. Srinivasan, "A Portrait of an ISO STEP Tolerancing Standard as an Enabler of Smart Manufacturing Systems," Journal of Computing and Information Science in Engineering, vol. 15, no. 2, pp. 021001-021001-5, 2015.

[22] A. Venkiteswaran, S. M. Hejazi, D. Biswas, J. J. Shah, and J. K. Davidson, "Semantic Interoperability of GD\&T Data Through ISO 10303 Step AP242," no. 50114, p. V02BT03A018, 2016.

[23] M. Mabkhot, A. Al-Ahmari, B. Salah, and H. Alkhalefah, "Requirements of the Smart Factory System: A Survey and Perspective," Machines, vol. 6, no. 2, p. 23, 2018.

[24] M. Rauch, R. Laguionie, J.-Y. Hascoet, and S.-H. Suh, "An advanced STEP-NC controller for intelligent machining processes," Robotics and Computer-Integrated Manufacturing, vol. 28, no. 3, pp. 375-384, 2012.

[25] S. H. Suh, B. E. Lee, D. H. Chung, and S. U. Cheon, "Architecture and implementation of a shop-floor programming system for STEP-compliant CNC," Computer-Aided Design, vol. 35, no. 12, pp. 1069-1083, 2003.
[26] J. Jasperneite, A. Neumann, and F. Pethig, "OPC UA versus MTConnect," Computer\&Automation (Sonderheft S2 2015 Control\&Drives) S, pp. 16-21, 2015.

[27] W. Sobel, "MTConnect standard. Part 1-overview and protocol," Standard-MTConnect. Available: http://www. mtconnect. org/standard. Accessed, vol. 10, 2015, [Accessed March 3, 2019].

[28] W. Mahnke, S.-H. Leitner, and M. Damm, OPC unified architecture. Springer Science \& Business Media, 2009.

[29] B. Edrington, B. Zhao, A. Hansel, M. Mori, and M. Fujishima, "Machine Monitoring System Based on MTConnect Technology," Procedia CIRP, vol. 22, pp. 92-97, 2014.

[30] J. Michaloski, B. E. Lee, F. Proctor, and S. Venkatesh, "Web-Enabled Real-Time Quality Feedback for Factory Systems Using MTConnect," in ASME 2012 International Design Engineering Technical Conferences and Computers and Information in Engineering Conference, 2012, pp. 403-409.

[31] N. Bengtsson, J. Michaloski, F. Proctor, G. Shao, and S. Venkatesh, "Towards data driven sustainable machining combining MTconnect production data and discrete event simulation," in Proceedings of the Proceedings of ASME 2010 International Manufacturing Science and Engineering Conference, 2010.

[32] S.-J. Shin, J. Woo, D. B. Kim, S. Kumaraguru, and S. Rachuri, "Developing a virtual machining model to generate MTConnect machine-monitoring data from STEP-NC," International Journal of Production Research, vol. 54, no. 15, pp. 4487-4505, 2016.

[33] X. F. Liu, M. R. Shahriar, S. M. N. A. Sunny, M. C. Leu, M. Cheng, and L. Hu, "Design and Implementation of Cyber-Physical Manufacturing Cloud Using MTConnect," no. 50084, p. V01BT02A019, 2016.

[34] Yueh-Ling Lin, Chih-Chieh Lin, and Hung-Sheng Chiu, "Developing a cloud virtual maintenance system for machine tools management," in 2015 11th International Conference on Heterogeneous Networking for Quality, Reliability, Security and Robustness (QSHINE), 2015, pp. 358-364.

[35] C. Liu, H. Vengayil, R. Y. Zhong, and X. Xu, "A systematic development method for cyber-physical machine tools," Journal of Manufacturing Systems, vol. 48, pp. 13-24, 2018.

[36] OPC UA, "Industrie 4.0-enabling technology with high diversity and variability Schleipen, M.; Gilani, S.-S.; Bischoff, T.; Pfrommer, J," in 49th CIRP Conference on Manufacturing Systems (CIRP-CMS 2016), CIRP, 2016

[37] I. Ayatollahi, B. Kittl, F. Pauker, and M. Hackhofer, "Prototype OPC UA server for remote control of machine tools," in Proceedings of International Conference on Innovative Technologies, 2013, pp. 73-76.

[38] J. Schlechtendahl, M. Keinert, F. Kretschmer, A. Lechler, and A. Verl, "Making existing production systems Industry 4.0-ready," Production Engineering, vol. 9, no. 1, pp. 143-148, 2015.

[39] C. Liu, X. Hong, Z. Zhu, and X. Xu, "Machine Tool Digital Twin: Modelling methodology and applications," presented at the Proceedings of International Conference on Computers and Industrial Engineering, CIE, 2018, vol. 2018-December.

[40] MTConnect Institute, OPC Foundation. "MTConnect-OPC UA Companion Specification," Available: https://static1.squarespace.com/static/54011775e 4b0bc1 fe0fb8494/t/5 581c564e4b0f8af8806da42/1434568036828/MTConnect-OPC UA C ompanion_Specification_v1.0.pdf [Accessed February 16, 2019].

[41] DMSC. Home - QIF Standard. Available: http://qifstandards.org/ [Accessed July 7, 2018].

[42] W. Z. Bernstein, T. D. Hedberg Jr, M. Helu, and A. B. Feeney, "Contextualising manufacturing data for lifecycle decision-making," International journal of product lifecycle management, vol. 10, no. 4, p. $326,2018$.

[43] F. Proctor, M. Franaszek, and J. Michaloski, "Tolerances and Uncertainty in Robotic Systems," no. 58356, p. V002T02A075, 2017.

[44] J. L. Michaloski, Y. F. Zhao, B. E. Lee, and W. G. Rippey, "Web-enabled, Real-time, Quality Assurance for Machining Production Systems," Procedia CIRP, vol. 10, pp. 332-339, 2013. 